Research Article

\title{
First-Principles Calculations of the Structure Stability and Mechanical Properties of LiFeAs and NaFeAs under Pressure
}

\author{
Zhengquan Hu $\mathbb{D},{ }^{1}$ Weiwei $X{ }^{2},{ }^{2}$ Cai Chen, ${ }^{3}$ Yufeng Wen, ${ }^{4,5}$ and Lili Liu ${ }^{6}{ }^{6}$ \\ ${ }^{1}$ College of Electronic and Information Engineering, Chongqing Three Gorges University, Chongqing 404100, China \\ ${ }^{2}$ Institute for Structure and Function, Chongqing University, Chongqing 401331, China \\ ${ }^{3}$ College of Civil Engineering, Chongqing Three Gorges University, Chongqing 404100, China \\ ${ }^{4}$ School of Mathematical Sciences and Physics, Jinggangshan University, Ji'an 343009, China \\ ${ }^{5}$ School of Materials Science and Engineering, Shanghai Jiao Tong University, Shanghai 200240, China \\ ${ }^{6}$ Department of Physics, Chongqing Three Gorges University, Chongqing 404100, China
}

Correspondence should be addressed to Zhengquan Hu; hzq250314@163.com

Received 13 September 2017; Revised 17 December 2017; Accepted 16 January 2018; Published 27 March 2018

Academic Editor: David Holec

Copyright (c) 2018 Zhengquan Hu et al. This is an open access article distributed under the Creative Commons Attribution License, which permits unrestricted use, distribution, and reproduction in any medium, provided the original work is properly cited.

The lattice parameters and elastic constants of the tetragonal LiFeAs and NaFeAs under different pressures have been investigated by using the first-principles calculations. It is found that their lattice parameters at $0 \mathrm{GPa}$ are in agreement with the available experimental data. By the elastic stability criteria under isotropic pressure, it is found that LiFeAs and NaFeAs with the tetragonal structure are not mechanically stable above $16 \mathrm{GPa}$ and $18 \mathrm{GPa}$, respectively. Besides, Pugh's modulus ratio, Poisson's ratio, Vickers hardness, and elastic anisotropy factors of LiFeAs in the pressure range of 0-16 GPa and NaFeAs in the pressure range of $0-18 \mathrm{GPa}$ are systematically investigated. It is shown that their ductilities increase with increasing pressure, and the ductility of $\mathrm{NaFeAs}$ is superior to that of LiFeAs under different pressures.

\section{Introduction}

The recent discovery of superconductivity in $\mathrm{LaO}_{1-x} \mathrm{~F}_{x} \mathrm{FeAs}$ with a superconducting transition temperature $\left(T_{c}\right)$ of $26 \mathrm{~K}$ has generated tremendous interest in the ion based on the layered superconductor field [1]. Substitution of lanthanum in $\mathrm{LaO}_{1-x} \mathrm{~F}_{x} \mathrm{FeAs}$ with other rare earth metals results in a series of superconducting compounds $\mathrm{REFeAsO}_{1-x} \mathrm{~F}_{x}$, where $\mathrm{RE}=\mathrm{Ce}, \mathrm{Pr}, \mathrm{Nd}, \mathrm{Sm}$, or $\mathrm{Cd}$, with $T_{c} s$ closing or exceeding $50 \mathrm{~K}$ [2-5]. Fe-based superconductors are a broad family of groups including three main types of Fe-based superconductors, namely, $\mathrm{REFeAsO}(\mathrm{RE}=$ rare earth, $\mathrm{ab}-$ breviated as 1111 type), $\mathrm{AeFe}_{2} \mathrm{As}_{2}(\mathrm{Ae}=$ alkaline earth, abbreviated as 122 type), and AFeAs ( $\mathrm{A}=$ alkaline, abbreviated as 111 type). Parent compounds of 1111 and 122 types do not superconduct, but 111 type compounds LiFeAs and NaFeAs superconduct [6]. The critical transition temperature values of LiFeAs and NaFeAs are $T_{c}=18 \mathrm{~K}$ and $T_{c}=9 \mathrm{~K}$, respectively [7-10]. The absence of the spin density wave (SDW) transition and the relatively low $T_{c}$ in comparison with the 1111 type and 122 type of iron-based superconductors make LiFeAs and $\mathrm{NaFeAs}$ possible candidates for being conventional BCS superconductors. In some sense, LiFeAs and NaFeAs are important with regard to understanding the mechanism of superconductivity in ironbased superconductors. The compounds LiFeAs and $\mathrm{NaFeAs}$ have a structure of tetragonal symmetry $(\mathrm{P} 4 / \mathrm{nmm})$, and $\alpha$-PbO-type layers of edge-sharing $\mathrm{FeAs}_{4 / 4}$ tetrahedra interspersed with $\mathrm{Li}$ and $\mathrm{Na}$ atoms at interstitial sites of the As layer, which is shown in Figure 1. However, owing to the evaporation loss of Li/Na during the high-temperature reaction [11], the stoichiometric LiFeAs and NaFeAs compounds are difficult to be synthesized experimentally. With the fast development of computer, various properties of LiFeAs and $\mathrm{NaFeAs}$ compounds have been investigated by the first-principles calculations. For example, the elastic coefficients, bulk moduli, and shear moduli of LiFeAs crystals have been calculated from the first principles using the full-potential linearized augmented plane wave (FLAPW) method [12]. Li et al. [13] investigated the crystal, 


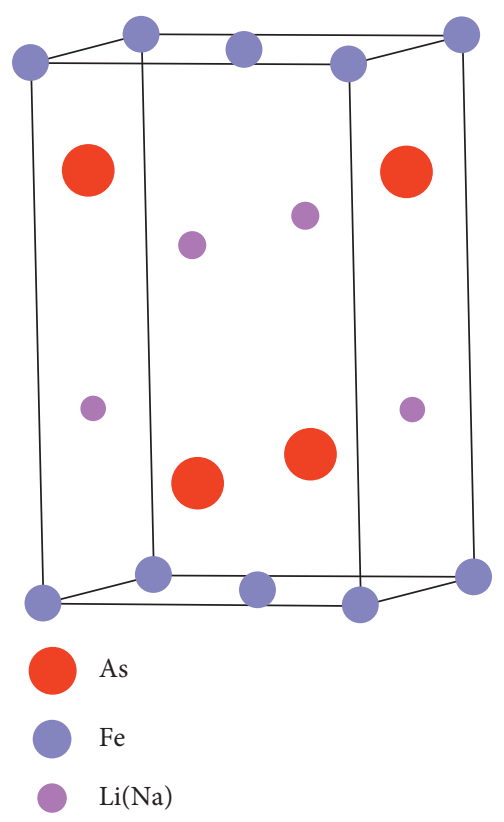

FIgURE 1: Crystal structure of $\operatorname{Li}(\mathrm{Na}) \mathrm{FeAs}$.

spin, and electronic structure of LiFeAs superconductors by the first-principles calculations based on the density functional theory. More recently, a study on doping effect on the electronic band structure and magnetic properties of LiFeAs and $\mathrm{NaFeAs}$ compounds was carried out using ab initio method [11]. These results are very important to further scientific and technical investigations.

Elastic properties of a solid are significant because some physical properties such as the equation of state, specific heat, thermal expansion, bulk modulus, Young's modulus, melting point, and Poisson's ratio can be determined from its elastic constants. The velocity of sound of the longitudinal wave and shear wave and the strength of a material can be also derived from its elastic constants [14]. However, to date, elastic constants of LiFeAs and $\mathrm{NaFeAs}$ under high pressures have never been fully investigated. Following this, the present study has been conducted to predict the elastic properties of LiFeAs and $\mathrm{NaFeAs}$ under high pressure by using the first-principles calculations within the density functional theory. In addition, various mechanical properties including the bulk modulus, shear modulus, Poisson's ratio, and elastic wave velocities have also been obtained and discussed in detail with increasing pressure based on the calculated elastic constants. The paper is organized as follows: the theoretical method is briefly described in Section 2; Section 3 presents the calculated results with some discussions; and Section 4 presents the conclusions.

\section{Method of Calculation}

In the present work, the first-principles calculations were performed by using the projector augmented wave (PAW) method $[15,16]$ within the generalized gradient approximation (GGA), as implemented in the VASP code developed at the Institute für Materialphysik of Universität Wien [1719]. The exchange-correlation effects were treated within the Perdew-Burke-Ernzerhof (PBE) approximation [20, 21]. In this work, $2 s^{1}, 2 p^{6} 3 s^{1}, 3 p^{6} 4 s^{1} 3 d^{7}$, and $4 s^{2} 4 p^{3}$ are treated as the valence electrons for $\mathrm{Li}, \mathrm{Na}, \mathrm{Fe}$, and As, respectively. The $k$ point meshes for Brillouin zone sampling were constructed using the Monkhorst-Pack scheme [22], and the $10 \times 10 \times 8 k$ point mesh is employed to calculate the lattice and elastic constants of LiFeAs and $\mathrm{NaFeAs}$ under different pressures. In addition, a plane-wave basis set with energy cutoff $500 \mathrm{eV}$ is used, and the energy convergence criterion is set to $10^{-6} \mathrm{eV}$ for all calculations. The structural parameters of LiFeAs and $\mathrm{NaFeAs}$ under different pressures are optimized by the full relaxations with respect to the volume, shape, and all internal atomic positions until the change in the total energy is smaller than $10^{-5} \mathrm{eV}$ between two ionic relaxation steps. LiFeAs and $\mathrm{NaFeAs}$ are detected to have no magnetic order at any temperature, so spin polarization is not considered in all calculations.

Let $x_{i}$ be the initial configuration of a material element, and the same material element has coordinate $x_{i}^{\prime}$ after a homogeneous elastic deformation. The deformation gradient is described as

$$
F_{i j}=\frac{\partial x_{i}^{\prime}}{\partial x_{j}}
$$

From the deformation gradients, we define finite Lagrangian strains as follows:

$$
\eta_{i j}=\frac{1}{2} \sum_{p=1}^{3}\left(F_{p i} F_{p j}-\delta_{i j}\right)
$$

The elastic energy $(\Delta E)$ can be expanded in a Taylor series in terms of the strain tensor as follows:

$$
\Delta E=\frac{V}{2 !} \sum_{i j k l} C_{i j k l} \eta_{i j} \eta_{k l}+\frac{V}{3 !} \sum_{i j k l m n} C_{i j k l m n} \eta_{i j} \eta_{k l} \eta_{m n},
$$

where $V$ is the volume of the unstrained lattice. After using the Voigt notation for the strain tensors $\eta_{11} \rightarrow \eta_{1}, \eta_{22} \rightarrow \eta_{2}$, $\eta_{33} \rightarrow \eta_{3}, \eta_{23} \rightarrow \eta_{4} / 2, \eta_{31} \rightarrow \eta_{5} / 2$, and $\eta_{12} \rightarrow \eta_{6} / 2$, (3) can be simply expressed as

$$
\Delta E=\frac{V}{2 !} \sum_{i, j=1}^{6} C_{i j} \eta_{i} \eta_{j}+\frac{V}{3 !} \sum_{i, j, k=1}^{6} C_{i j k} \eta_{i} \eta_{j} \eta_{k}
$$

For LiFeAs and $\mathrm{NaFeAs}$ with the tetragonal structure, there are six independent elastic constants $C_{11}, C_{12}, C_{13}, C_{33}$, $C_{44}$, and $C_{66}$. To obtain the complete set of the elastic constants, we introduce six Lagrangian strain tensors in terms of a single strain parameter $\xi$. The elastic energy per unit mass can be written as an expansion in the strain parameter $\xi$ :

$$
\Phi=\frac{\Delta E}{V}=\frac{1}{2} \Lambda_{2} \xi^{2}+O\left(\xi^{3}\right) .
$$

The coefficient $\Lambda_{2}$ is combinations of the elastic constants $\left(C_{11}, C_{12}, C_{13}, C_{33}, C_{44}\right.$, and $\left.C_{66}\right)$. The six specific strain tensors labeled as $N_{\alpha}(\alpha=1,2, \ldots, 6)$ can be expressed as 


$$
\begin{aligned}
& N_{1}=\left(\begin{array}{lll}
\xi & 0 & 0 \\
0 & 0 & 0 \\
0 & 0 & 0
\end{array}\right), \\
& N_{2}=\left(\begin{array}{lll}
\xi & 0 & 0 \\
0 & \xi & 0 \\
0 & 0 & 0
\end{array}\right), \\
& N_{3}=\left(\begin{array}{lll}
0 & 0 & 0 \\
0 & 0 & 0 \\
0 & 0 & \xi
\end{array}\right), \\
& N_{4}=\left(\begin{array}{lll}
\xi & 0 & 0 \\
0 & \xi & 0 \\
0 & 0 & \xi
\end{array}\right), \\
& N_{5}=\left(\begin{array}{lll}
\xi & 0 & 0 \\
0 & 0 & \xi \\
0 & \xi & 0
\end{array}\right), \\
& N_{6}=\left(\begin{array}{lll}
\xi & \xi & 0 \\
\xi & 0 & 0 \\
0 & 0 & 0
\end{array}\right) .
\end{aligned}
$$

The corresponding strain energy density can be expressed as

$$
\begin{aligned}
& \Delta E \frac{\left(N_{1}\right)}{V}=\frac{1}{2} C_{11} \xi^{2}+O\left(\xi^{3}\right), \\
& \Delta E \frac{\left(N_{2}\right)}{V}=\left(C_{11}+C_{12}\right) \xi^{2}+O\left(\xi^{3}\right), \\
& \Delta E \frac{\left(N_{3}\right)}{V}=\frac{1}{2} C_{33} \xi^{2}+O\left(\xi^{3}\right), \\
& \Delta E \frac{\left(N_{4}\right)}{V}=\frac{1}{2}\left(2 C_{11}+2 C_{12}+4 C_{13}+C_{33}\right) \xi^{2}+O\left(\xi^{3}\right), \\
& \Delta E \frac{\left(N_{5}\right)}{V}=\frac{1}{2}\left(C_{11}+4 C_{44}\right) \xi^{2}+O\left(\xi^{3}\right), \\
& \Delta E \frac{\left(N_{6}\right)}{V}=\frac{1}{2}\left(C_{11}+4 C_{66}\right) \xi^{2}+O\left(\xi^{3}\right) .
\end{aligned}
$$

For each strain tensor $N_{\alpha}$, the strain parameter $\xi$ varies from -0.02 to 0.02 with a finite step size of 0.002 .

Based on the calculated elastic constants, the bulk modulus $B$ and shear modulus $G$ can be evaluated in terms of the Voigt-Reuss-Hill (VRH) scheme [23]. The Voigt bounds on bulk $\left(B_{\mathrm{V}}\right)$ and shear $\left(G_{\mathrm{V}}\right)$ moduli are defined as [24]

$$
\begin{aligned}
B_{\mathrm{V}} & =\frac{1}{9}\left[2\left(C_{11}+C_{12}\right)+C_{33}+4 C_{13}\right], \\
G_{\mathrm{V}} & =\frac{1}{30}\left(M+3 C_{11}-3 C_{12}+12 C_{44}+6 C_{66}\right), \\
M & =C_{11}+C_{12}+2 C_{33}-4 C_{13},
\end{aligned}
$$

and the Reuss bounds on bulk $\left(B_{\mathrm{R}}\right)$ and shear $\left(G_{\mathrm{R}}\right)$ moduli are defined as [25]
TABLe 1: Calculated lattice constants ( $a$ and $c$ in $\AA$ ) and primitive cell volume $\left(V\right.$ in $\left.\AA^{3}\right)$ for tetragonal LiFeAs and NaFeAs under different pressures $(P$ in $\mathrm{GPa})$ at $0 \mathrm{~K}$.

\begin{tabular}{lcccccc}
\hline \multirow{P}{*}{$P$} & \multicolumn{3}{c}{ LiFeAs } & \multicolumn{3}{c}{ NaFeAs } \\
& $a$ & $c$ & $V$ & $a$ & $c$ & $V$ \\
\hline 0 & 3.791 & 6.112 & 87.840 & 3.929 & 6.890 & 106.361 \\
5 & 3.718 & 5.949 & 82.236 & 3.858 & 6.610 & 98.384 \\
10 & 3.660 & 5.835 & 78.163 & 3.795 & 6.466 & 93.123 \\
15 & 3.614 & 5.741 & 74.983 & 3.742 & 6.361 & 89.070 \\
16 & 3.605 & 5.723 & 74.376 & 3.732 & 6.344 & 88.358 \\
17 & 3.597 & 5.707 & 73.839 & 3.723 & 6.327 & 87.697 \\
18 & - & - & - & 3.714 & 6.311 & 87.053 \\
19 & - & - & - & 3.705 & 6.295 & 86.412 \\
\hline
\end{tabular}

$$
\begin{aligned}
B_{\mathrm{R}} & =\frac{C^{2}}{M}, \\
G_{\mathrm{R}} & =\frac{15}{\left(\left(18 B_{\mathrm{V}}\right) / C^{2}+6 /\left(C_{11}-C_{12}\right)+6 / C_{44}+3 / C_{66}\right)}, \\
C^{2} & =\left(C_{11}+C_{12}\right) C_{33}-2 C_{13}^{2} .
\end{aligned}
$$

The Hill averages of bulk $(B)$ and shear $(G)$ moduli are obtained by

$$
\begin{gathered}
B=\frac{\left(B_{\mathrm{V}}+B_{\mathrm{R}}\right)}{2}, \\
G=\frac{\left(G_{\mathrm{V}}+G_{\mathrm{R}}\right)}{2} .
\end{gathered}
$$

\section{Results and Discussion}

In Table 1, we list the lattice structures of LiFeAs and $\mathrm{NaFeAs}$ with the tetragonal structure under different pressures. The optimized lattice parameters at zero pressure are $a=3.791 \AA$, $c=6.113 \AA$ and $a=3.929 \AA, c=6.902 \AA$ for LiFeAs and $\mathrm{NaFeAs}$, respectively, which agree with the available experimental values $a=3.769 \AA, c=6.306 \AA$ for LiFeAs and $a=3.947 \AA, c=6.991 \AA$ for NaFeAs [7-10] and the theoretical values $a=3.77 \AA, c=6.10 \AA$ for LiFeAs and $a=3.89 \AA$, $c=6.87 \AA$ for NaFeAs [26]. It is a pity that there are no experimental data under high pressure for comparison. It is found that the lattice parameters decrease with increasing pressure. Besides, the lattice parameters and the volume of $\mathrm{NaFeAs}$ are always larger than those of LiFeAs under different pressures, which can be easily explained by the fact that the atomic radius of $\mathrm{Li}$ is smaller than that of $\mathrm{Na}$.

Knowledge of the elastic constants is crucial for understanding the structural stability, the anisotropic character of the bonding, and the bonding characteristic between the adjacent atomic planes. The present results $\left(C_{11}=144.9 \mathrm{GPa}, C_{12}=37.1 \mathrm{GPa}, C_{13}=35.3 \mathrm{GPa}, C_{33}=101.8 \mathrm{GPa}\right.$, $C_{44}=27.3 \mathrm{GPa}$, and $C_{66}=63.8 \mathrm{GPa}$ for $\mathrm{LiFeAs}$ and $C_{11}=117.4 \mathrm{GPa}, C_{12}=36.7 \mathrm{GPa}, C_{13}=30.0 \mathrm{GPa}, C_{33}=54.8 \mathrm{GPa}$, $C_{44}=25.9 \mathrm{GPa}$, and $C_{66}=58.1 \mathrm{GPa}$ for $\mathrm{NaFeAs}$ ) nearly agree with 


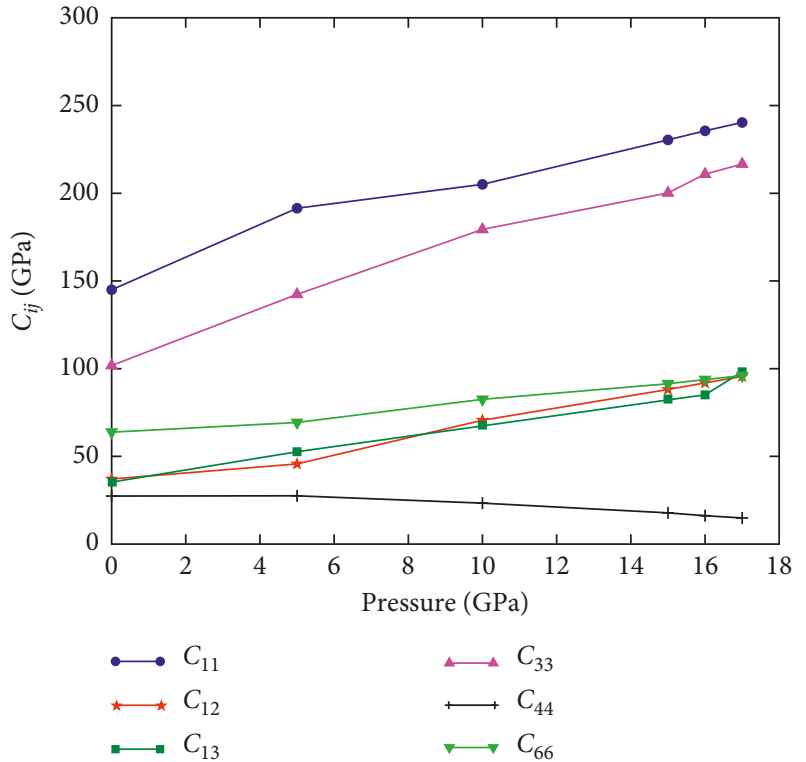

(a)

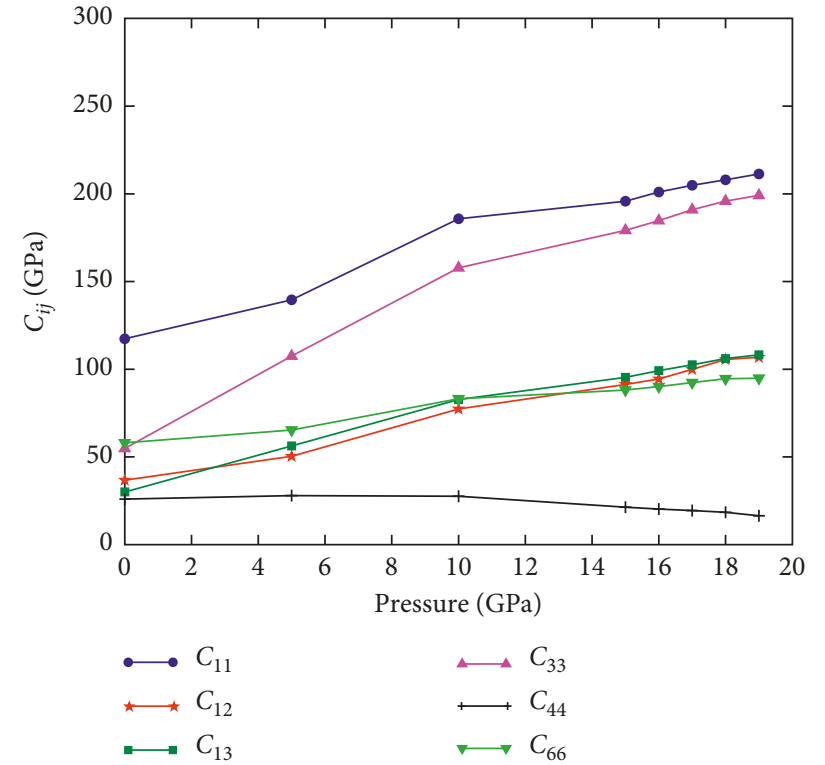

(b)

FIgURE 2: The pressure dependence of the elastic constants for (a) LiFeAs and (b) NaFeAs, respectively.

the recent theoretical results $\left(C_{11}=180.5 \mathrm{GPa}, C_{12}=56.0 \mathrm{GPa}\right.$, $C_{13}=60.5 \mathrm{GPa}, C_{33}=104.4 \mathrm{GPa}, C_{44}=52.1 \mathrm{GPa}$, and $C_{66}=$ 66.9 GPa for LiFeAs and $C_{11}=147.8 \mathrm{GPa}, C_{12}=58.3 \mathrm{GPa}, C_{13}=$ $56.7 \mathrm{GPa}, C_{33}=110.3 \mathrm{GPa}, C_{44}=40.9 \mathrm{GPa}$, and $C_{66}=59.9 \mathrm{GPa}$ for $\mathrm{NaFeAs)}$ [26]. The pressure dependence of the elastic constants $\left(C_{11}, C_{12}, C_{13}, C_{33}, C_{44}\right.$, and $\left.C_{66}\right)$ of LiFeAs and NaFeAs is illustrated in Figure 2. The elastic constants $C_{11}$ and $C_{33}$ are closely associated with the uniaxial sound propagation, and the other elastic constants $C_{12}, C_{13}, C_{44}$, and $C_{66}$ are mainly related to the nonaxial sound propagation. From Figure 2, it can be seen that $C_{11}, C_{12}, C_{13}, C_{33}$, and $C_{66}$ increase with increasing pressure; however, $C_{44}$ has the opposite change trend. It is also noted from Figure 2 that the values of $C_{11}$ and $C_{33}$ are the largest among all the elastic constants under different pressures, which demonstrates that both LiFeAs and $\mathrm{NaFeAs}$ cannot be easily compressed under uniaxial stress. Furthermore, in both systems, $C_{11}>C_{33}$ is proved to be true in the whole range of pressure, which indicates that the atomic bonds along the [100] direction between the nearest neighbors are stronger than those along the [001] direction.

For a tetragonal crystal, the mechanical stability under isotropic pressure leads to restrictions on the elastic constants as follows [27-29]:

$$
\begin{aligned}
\widetilde{C}_{11}+\widetilde{C}_{33}-2 \widetilde{C}_{13}>0 \\
\widetilde{C}_{11}-\widetilde{C}_{12}>0 \\
\widetilde{C}_{i i}>0, \\
2 \widetilde{C}_{11}+\widetilde{C}_{33}+2 \widetilde{C}_{12}+4 \widetilde{C}_{13}>0,
\end{aligned}
$$

where $\widetilde{C}_{\alpha \alpha}=C_{\alpha \alpha}-P \quad(\alpha=1,3,4,6), \quad \widetilde{C}_{12}=C_{12}+P$, and $\widetilde{C}_{13}=C_{13}+P . \widetilde{C}_{44}^{P}>0$ cannot be firstly satisfied at higher pressure among all the mechanical stability conditions for

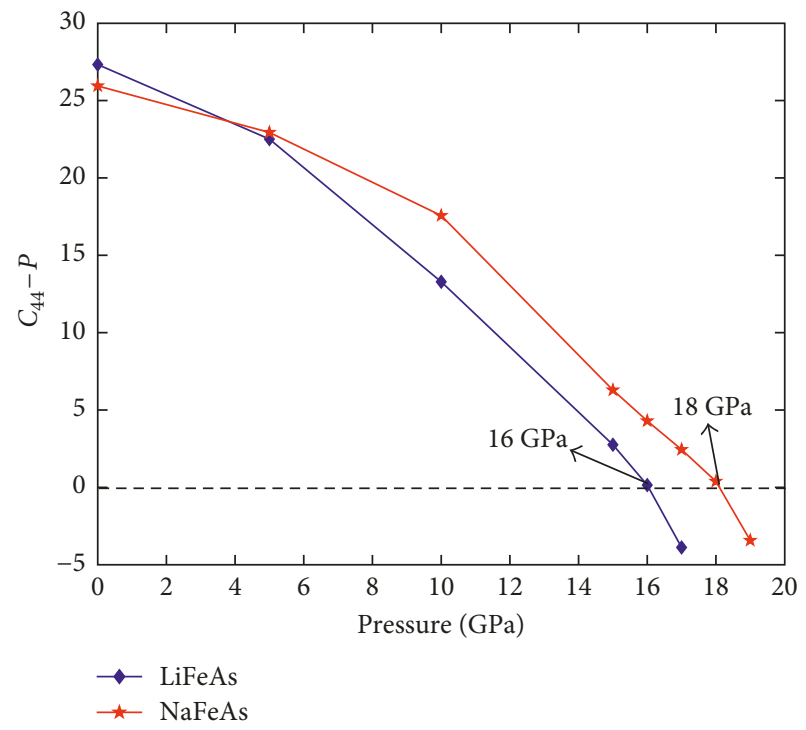

Figure 3: The $C_{44}-P$ as a function of pressure for LiFeAs and NaFeAs.

LiFeAs and NaFeAs. Figure 3 shows the pressure-dependent $C_{44}-P$ of LiFeAs and NaFeAs. When $P>16 \mathrm{GPa}$ for LiFeAs and $P>18 \mathrm{GPa}$ for NaFeAs, $C_{44}-P<0$, which suggests that their structure phase transitions may happen above pressures about $16 \mathrm{GPa}$ and $18 \mathrm{GPa}$ for LiFeAs and NaFeAs, respectively. The crystal structural evolution of $\mathrm{NaFeAs}$ as a function of pressure has been investigated by Liu et al. [30] through the synchrotron X-ray powder diffraction and diamond anvil cell technique, and a clear structural phase transition at about $20 \mathrm{GPa}$ is found. Subsequently, the pressure-induced structural phase transitions in LiFeAs and $\mathrm{NaFeAs}$ have been systematically investigated by Zhang et al. 


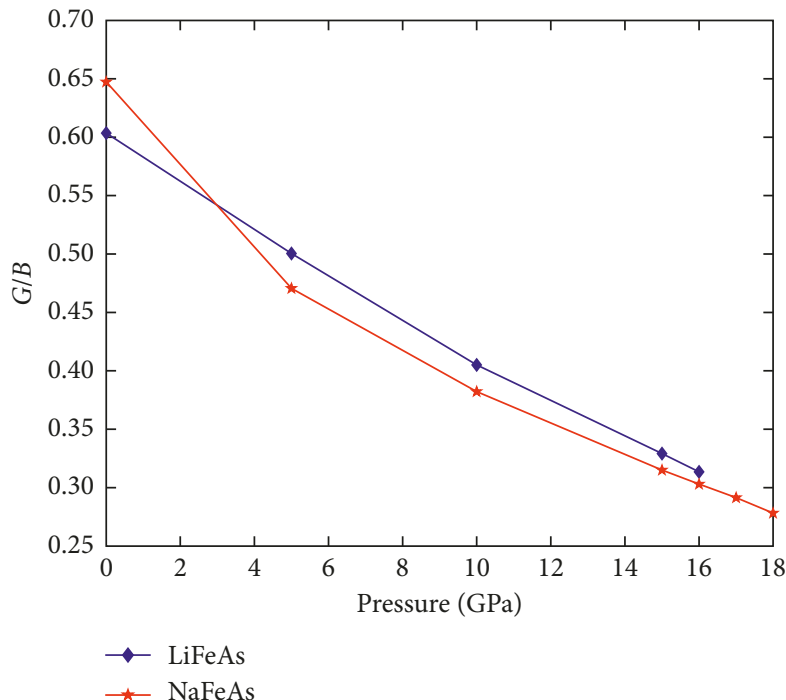

(a)

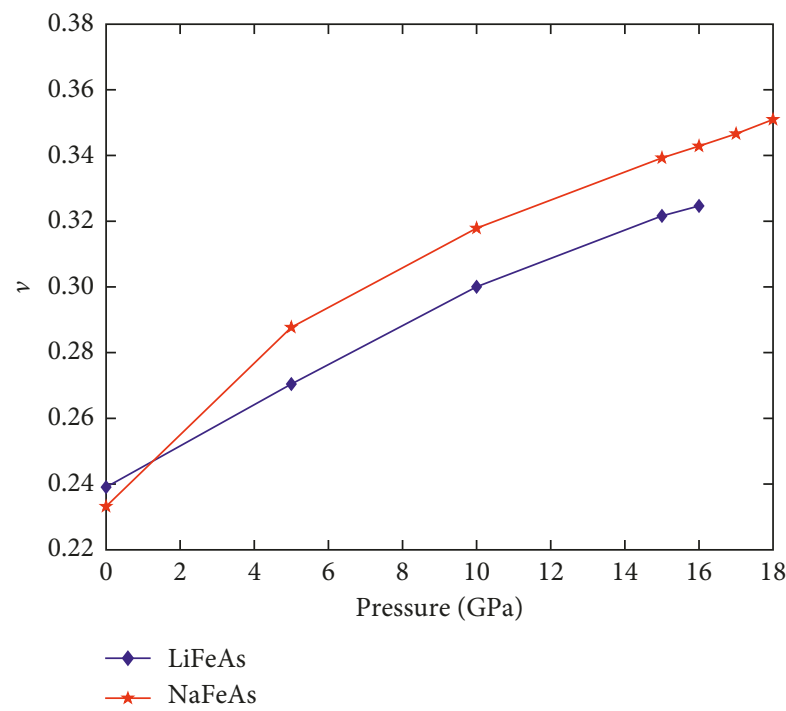

(c)
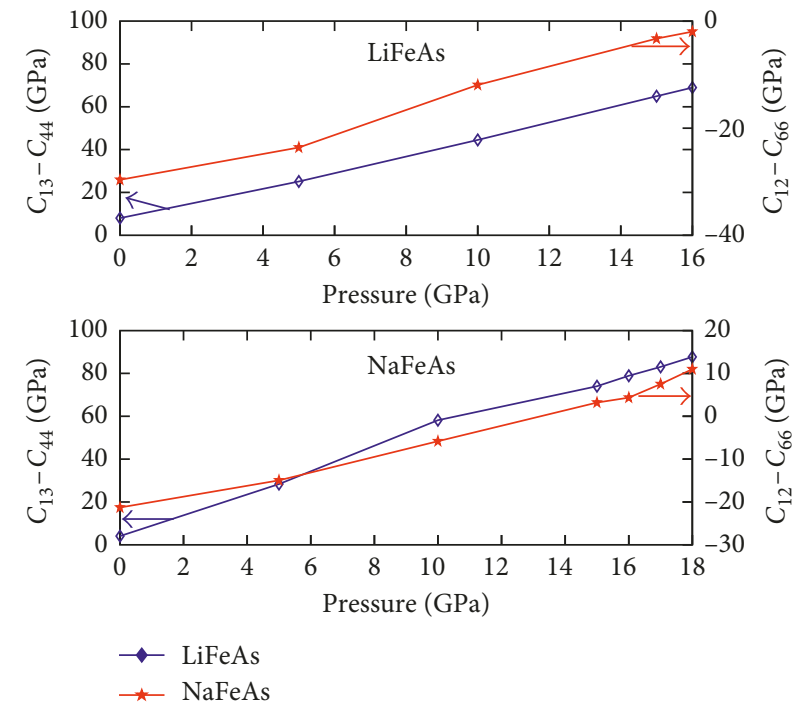

(b)

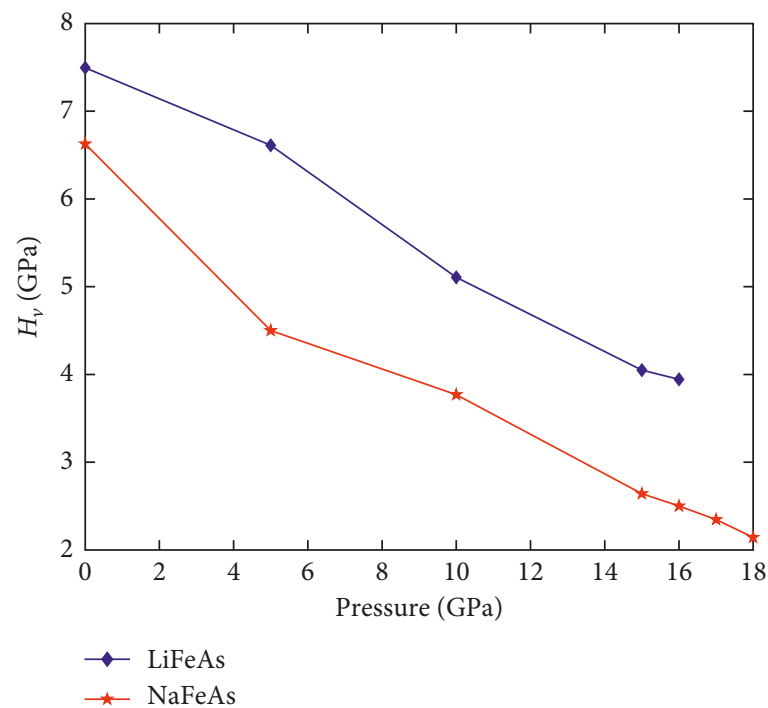

(d)

Figure 4: (a) The quotient of shear to bulk modulus $G / B$, (b) the Cauchy pressure, (c) Poisson's ratio $\nu$, and (d) the Vickers hardness $H_{v}$ versus pressure for $\mathrm{LiFeAs}$ and $\mathrm{NaFeAs}$.

[31] through the first-principles calculations based on the CALYPSO structural searches. And they reported that the structural phase transition happened at about $22 \mathrm{GPa}$ for $\mathrm{NaFeAs}$ and at about $22.2 \mathrm{GPa}$ for LiFeAs. Therefore, our results about the phase transition nearly agree with the previous experimental and theoretical values to a certain extent, especially for $\mathrm{NaFeAs}$.

In order to predict the plastic properties of a solid, a simple relationship predicted by Pugh [32] links empirically the brittle or ductile behavior of materials with their elastic moduli by $G / B$. The values of $B$ are $66.5 \mathrm{GPa}$ and $49.9 \mathrm{GPa}$ for LiFeAs and $\mathrm{NaFeAs}$, respectively, which are in agreement with the experimental values 57.3(6) GPa [33] and 52.3(2) GPa [33] for LiFeAs and NaFeAs, respectively. Meanwhile, the agreement between our calculations and experimental results at $0 \mathrm{GPa}$ provides reliability for the elastic properties under higher pressures. A high (low) $G / B$ value is associated with brittleness (ductility). If $G / B<0.57$, the material will display a ductile behavior; otherwise, it will display a brittle behavior. Figure $4(a)$ displays $G / B$ as a function of pressure. Obviously, the $G / B$ ratio decreases with increasing pressure, and the decreasing degree of $\mathrm{NaFeAs}$ is larger than that of LiFeAs in the whole pressure, which demonstrates that high pressure can improve their ductilities and more effectively improve the ductility of $\mathrm{NaFeAs}$. Besides, it is observed from Figure 4(a) that the $G / B$ value of $\mathrm{NaFeAs}$ is smaller than that of LiFeAs in the whole pressure in addition to the existing small difference at $0 \mathrm{GPa}$, illustrating that $\mathrm{NaFeAs}$ is more ductile than $\mathrm{LiFeAs}$ under different pressures except at $0 \mathrm{GPa}$. Pettifor has suggested that the angular character of atomic bonding in metals and intermetallics, which also relates to the brittle or ductile 


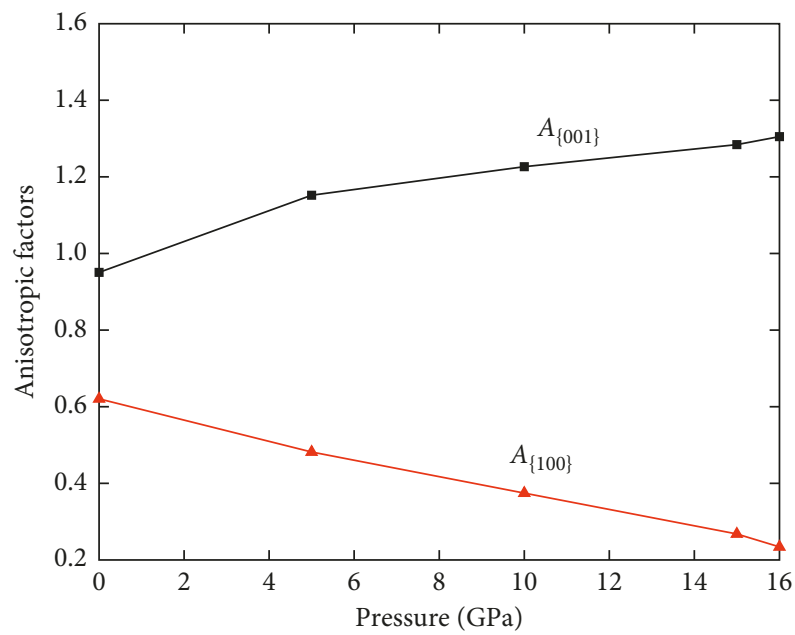

(a)

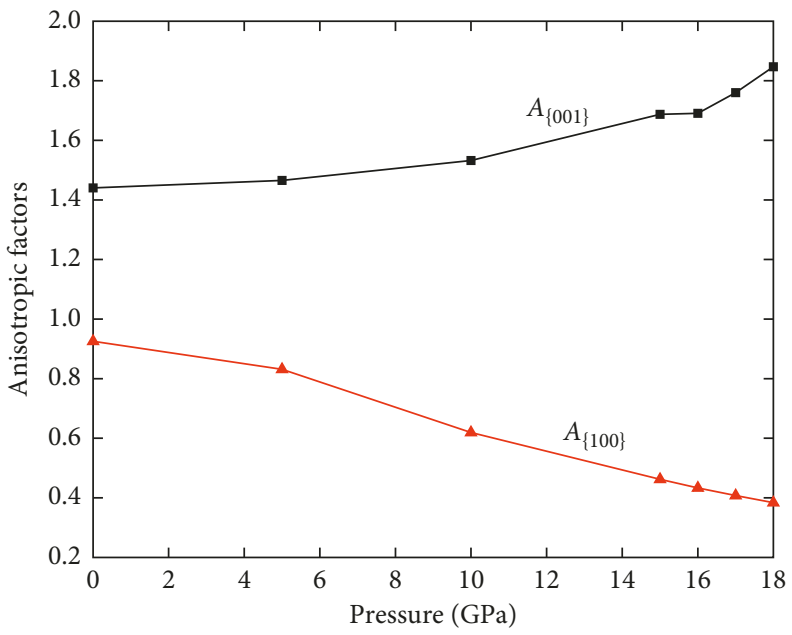

(b)

Figure 5: The pressure dependence of the anisotropic factors for (a) LiFeAs and (b) NaFeAs.

characteristics, could be described by the Cauchy pressure. For a tetragonal crystal, the Cauchy pressure can be defined as $C_{13}-C_{44}$ and $C_{12}-C_{66}$ [34]. For metallic bonding, the Cauchy pressure is typically positive, while for directional bonding, it is negative, with larger negative pressure representing a more directional character. The pressure dependence of Cauchy pressures of LiFeAs and NaFeAs is plotted in Figure 4(b). Obviously, their Cauchy pressures increase with increasing pressure, and NaFeAs has higher Cauchy pressure than LiFeAs under different pressures. That is, we can obtain the same conclusion from both the $G / B$ ratio and the Cauchy pressure. In addition, Poisson's ratio with $v=3 B-2 G /(2(3 B+G))$ can also reflect the ductile properties, which usually ranges from -1 to 0.5 . The higher the Poisson ratio is, the better the ductility is. Furthermore, $v$ is larger than $1 / 3$ for ductile materials and less than $1 / 3$ for brittle materials. Figure 4(c) presents the pressure dependence of Poisson's ratio for LiFeAs and NaFeAs. It is clear that, in both systems, the values of $v$ increase with increasing pressure, and the increasing degree of $\mathrm{NaFeAs}$ is larger than that of LiFeAs in the whole pressure. Moreover, hardness defined as the resistance of a material to deform is another important parameter to describe the mechanical properties of a material. As we all know, both Pugh's modulus ratio and Poisson's ratio have a close relationship with the hardness of a material. On the basis of Teter's famous empirical correlation [35], a simplified formula of Vickers hardness proposed by Chen et al. [36, 37] can be written as $H_{v}=2\left(k^{2} G\right)^{0.585}-3$, where $k=G / B$. This formula demonstrates that the hardness is not only related to the shear modulus but also to the bulk modulus. Figure 4(d) displays the change in Vickers hardness versus pressure for $\mathrm{LiFeAs}$ and $\mathrm{NaFeAs}$. It is noteworthy that the hardness data are intrinsic, ignoring microstructural effects. Obviously, in both systems, the hardness decreases with pressure, indicating that high pressure is conducive to the formation of ionic bonds and consequently results in the increase of ductility. Besides, NaFeAs has smaller Vickers hardness than
LiFeAs under different pressures, indicating that the metallic bonding of $\mathrm{NaFeAs}$ is stronger than that of LiFeAs, that is, the ductility of NaFeAs is superior to that of LiFeAs under different pressures.

The anisotropy is also a key parameter to determine the mechanical properties of a material. A proper description of anisotropic behavior has a significant implication in engineering science as well as in crystal physics. For a tetragonal crystal, the shear anisotropic factors along $\{100\}$ and $\{001\}$ planes are defined as follows $[14,28]$ :

$$
\begin{aligned}
& A_{1}=A_{\{100\}}=\frac{4 C_{44}}{C_{11}+C_{33}-2 C_{13}}, \\
& A_{2}=A_{\{001\}}=\frac{2 C_{66}}{C_{11}-C_{12}} .
\end{aligned}
$$

Both $A_{1}$ and $A_{2}$ must be equal to one for an isotropy crystal, while any value smaller or greater than one measures the degree of elastic anisotropy. The anisotropic factors $A_{1}$ and $A_{2}$ with pressure for LiFeAs and $\mathrm{NaFeAs}$ are plotted in Figure 5 . It can be found that, in both systems, $A_{\{100\}}$ decreases and $A_{\{001\}}$ increases with increasing pressure, which have the same change trend as that of the reported tetragonal $\mathrm{WN}_{2}$ [28]. $A_{\{100\}}$ decreases with pressure which may be resulted from the increase in the longitudinal modes $C_{11}$ and $C_{33}$ and decrease in the shear mode $C_{44}$ with pressure. Furthermore, it can be seen from Figure 5 that all the values of $A_{100}$ are smaller than one and those of $A_{001}$ are greater than one, that is, all the values deviate from one and the degree of deviation increases with increasing pressure. Therefore, it can be concluded that high pressure can make LiFeAs and NaFeAs more elastically anisotropic.

To elucidate the nature of the pressure effect, the total and partial density of states (DOSs) of LiFeAs and NaFeAs under different pressures are calculated, just as shown in Figures 6(a)-6(f). For LiFeAs, our calculated DOS at the ground state $(0 \mathrm{~K}$ and $0 \mathrm{GPa})$ is similar to that reported earlier [38, 39]. The DOS plots of LiFeAs and NaFeAs show 


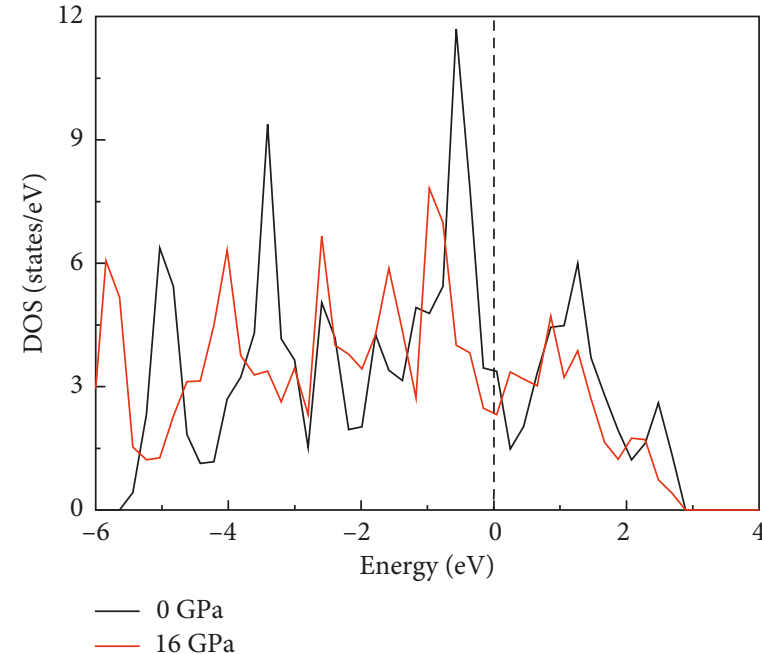

(a)

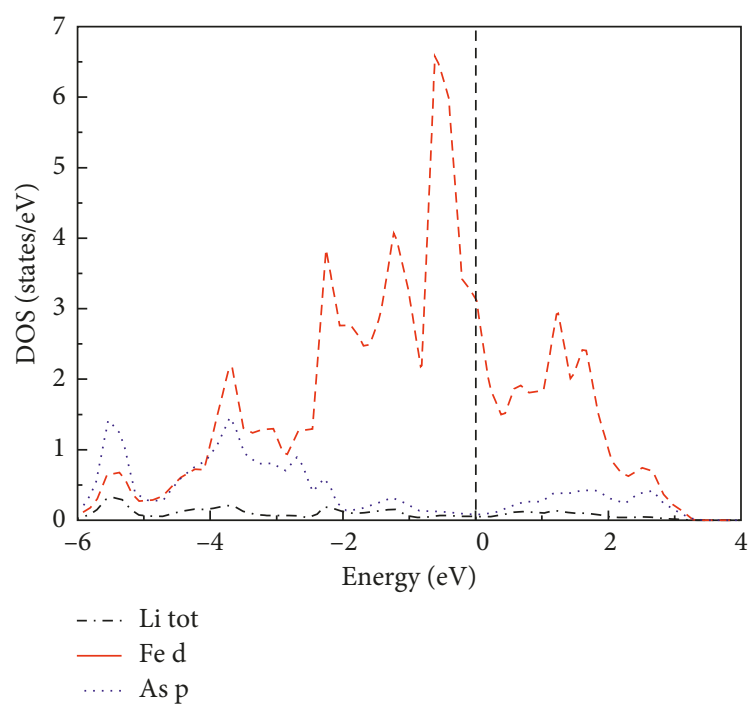

(c)

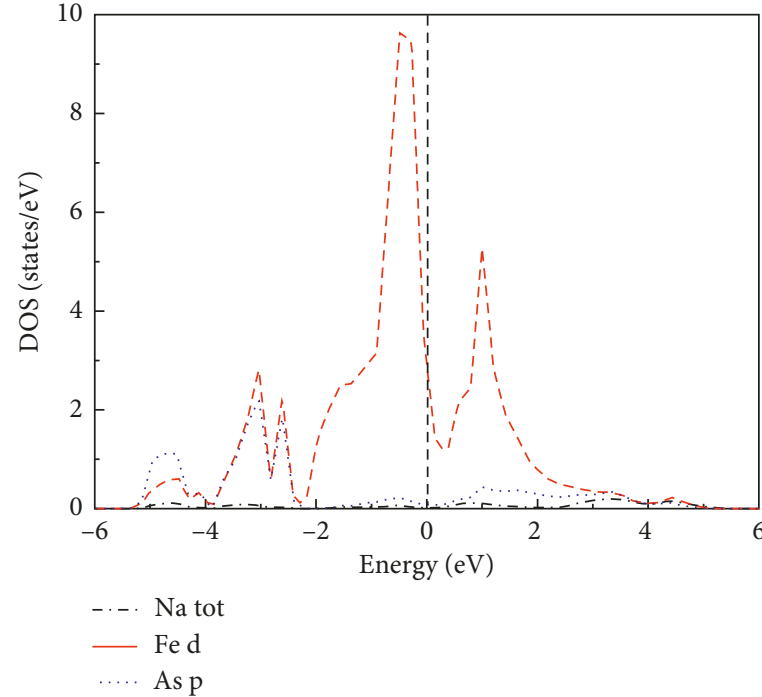

(e)

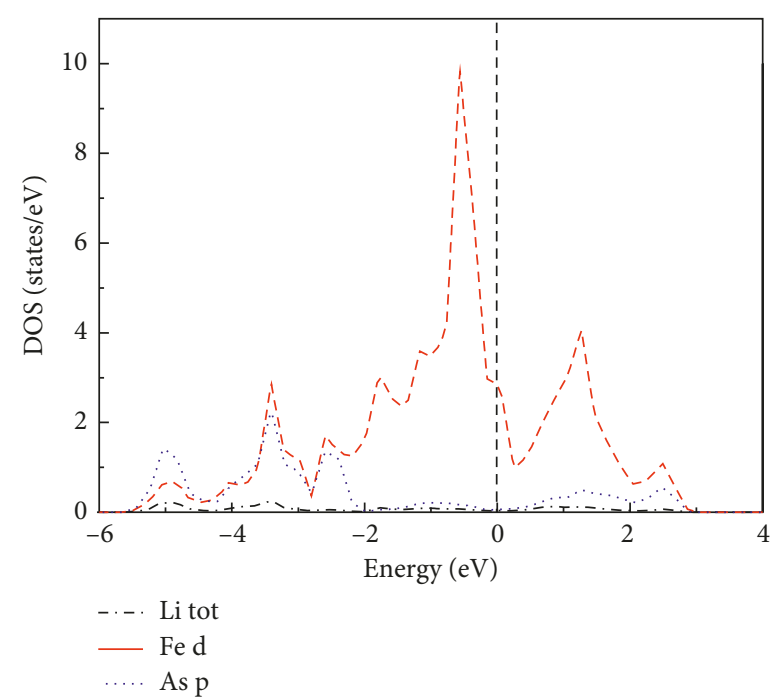

(b)

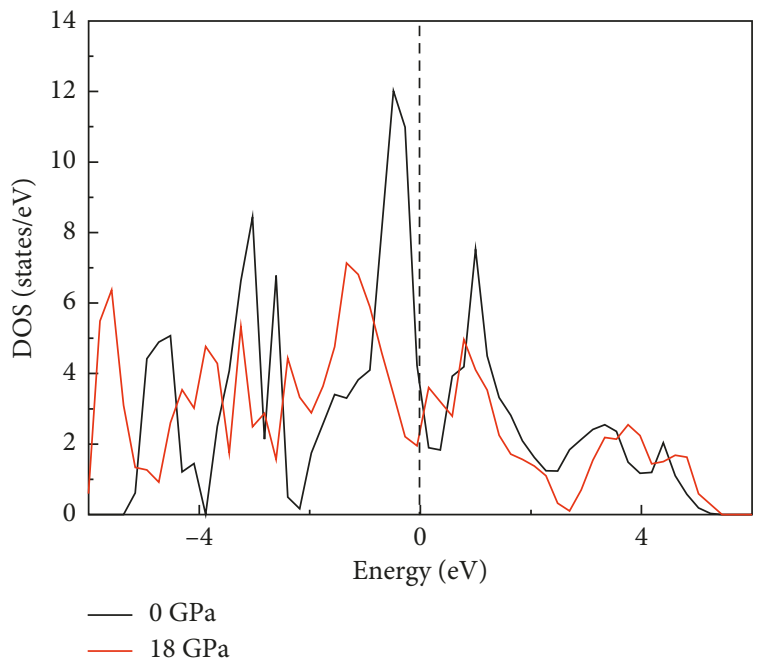

(d)

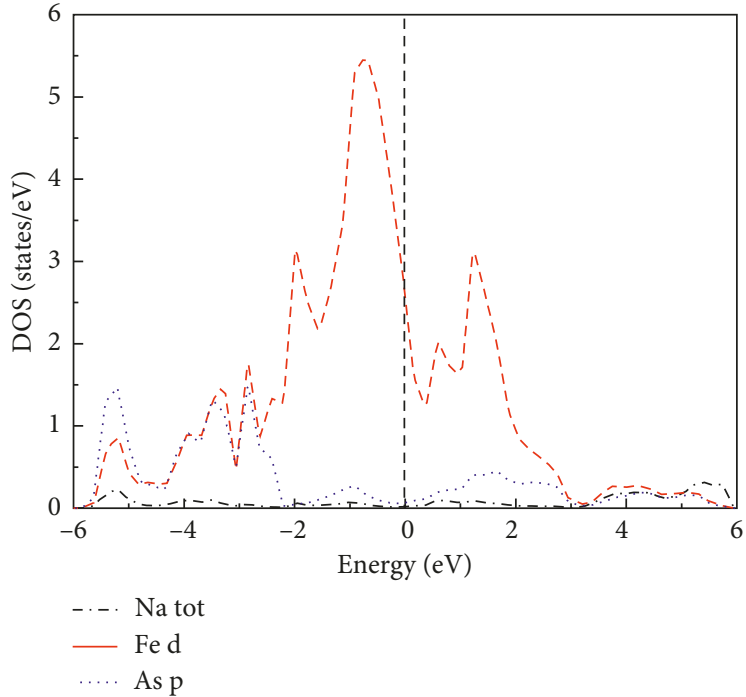

(f)

FIGURE 6: Density of states (DOSs) for LiFeAs (a) at $0 \mathrm{GPa}$ (b) and $16 \mathrm{GPa}$ (c) and NaFeAs (d) at $0 \mathrm{GPa}(\mathrm{e})$ and $18 \mathrm{GPa}$ (f). Both the total and atomic DOSs are shown. 
some generic features that are common to the parent compounds of the iron-based superconductors. That is, the total DOS is dominated by the Fe $3 \mathrm{~d}$ states in the vicinity of the Fermi energy, with only a small contribution from the As and alkali metal states and that is strongly decreasing with energy near the Fermi energy. Because the two compounds show similar features, we take LiFeAs as an example to analyze the pressure effect. From Figure 6(b), it can be seen that the substantial overlap between Fe $3 \mathrm{~d}$ and As $4 \mathrm{p}$ orbitals indicating the strong covalence of LiFeAs mainly comes from the orbital hybridization between Fe $3 d$ and As $4 p$ states from $-6 \mathrm{eV}$ to $-2 \mathrm{eV}$. When the external pressure is increased to $16 \mathrm{GPa}$, it can be seen from Figure 6(c) that the external pressure weakens the interaction of $\mathrm{Fe} 3 \mathrm{~d}$ and $\mathrm{As} 4 \mathrm{p}$, which indicates that higher pressure can enhance the ductility of LiFeAs.

\section{Conclusions}

In summary, the structure stability and mechanical properties of LiFeAs and NaFeAs under different pressures have been investigated by using the first-principles calculations. The pressure dependence of the lattice parameters and elastic constants of LiFeAs and $\mathrm{NaFeAs}$ are also presented. It is found that their lattice parameters at $0 \mathrm{GPa}$ are in agreement with the available experimental data. On the basis of the elastic constants under different pressures, the phase transitions of LiFeAs and $\mathrm{NaFeAs}$ are researched by the elastic stability criteria under isotropic pressure. It is predicted that $\mathrm{LiFeAs}$ and $\mathrm{NaFeAs}$ with the tetragonal structure are not mechanically stable above $16 \mathrm{GPa}$ and $18 \mathrm{GPa}$, respectively, which agree well with the previous experimental and theoretical values to a certain extent, especially for $\mathrm{NaFeAs}$. Besides, Pugh's modulus ratio $G / B$, Poisson's ratio $\nu$, the Vickers hardness $H_{v}$, and the elastic anisotropy of LiFeAs in the pressure range of $0-16 \mathrm{GPa}$ and NaFeAs in the pressure range of $0-18 \mathrm{GPa}$ are further investigated. It is shown that, in both systems, the ductilities increase with increasing pressure, and NaFeAs has more ductility than LiFeAs under different pressures. Besides, in both systems, $A_{\{100\}}$ decreases and $A_{\{001\}}$ increases with pressure, which have the same change trend as that of the reported tetragonal $\mathrm{WN}_{2}$.

\section{Conflicts of Interest}

The authors declare that they have no conflicts of interest.

\section{Authors' Contributions}

Lili Liu and Cai Chen carried out the theoretical calculations, Weiwei $\mathrm{Xu}$ analyzed the data, and Zhengquan $\mathrm{Hu}$ and Yufeng Wen wrote the paper.

\section{Acknowledgments}

This work was supported by the Program for Innovation Team Building at Institutions of Higher Education in Chongqing (CXTDX201601034).

\section{References}

[1] Y. Kamihara, T. Watanabe, M. Hirano, and H. Hosono, "Ironbased layered superconductor $\mathrm{La}\left[\mathrm{O}_{1-x} \mathrm{~F}_{x}\right] \mathrm{FeAs}(x=0.05-0.12)$ with $T_{\mathrm{c}}=26 \mathrm{~K}$," Journal of the American Chemical Society, vol. 130, no. 11, pp. 3296-3297, 2008.

[2] G. F. Chen, Z. Li, D. Wu et al., "Superconductivity at $41 \mathrm{~K}$ and its competition with spin-density-wave instability in layered $\mathrm{CeO}_{1-x} \mathrm{~F}_{x} \mathrm{FeAs}$," Physical Review Letters, vol. 100, no. 24, p. 247002, 2008.

[3] Z. A. Ren, J. Yang, W. Lu et al., "Superconductivity in the ironbased F-doped layered quaternary compound $\mathrm{Nd}\left[\begin{array}{ll}\mathrm{O}_{1-x} & \mathrm{~F}_{x}\end{array}\right]$ FeAs," Europhysics Letters, vol. 82, no. 5, p. 57002, 2008.

[4] X. H. Chen, T. Wu, G. Wu, R. H. Liu, H. Chen, and D. F. Fang, "Superconductivity at $43 \mathrm{~K}$ in $\mathrm{SmFeAsO}_{1-x} \mathrm{~F}_{x}$ " Nature, vol. 453, no. 7196, p. 761, 2008.

[5] P. Cheng, L. Fang, H. Yang et al., "Superconductivity at $36 \mathrm{~K}$ in gadolinium-arsenide oxides $\mathrm{GdO}_{1-x} \mathrm{~F}_{x}$ FeAs," Science in China Series G: Physics, Mechanics and Astronomy, vol. 51, no. 6, pp. 719-722, 2008.

[6] C. W. Chu, F. Chen, M. Gooch et al., "The synthesis and characterization of LiFeAs and NaFeAs," Physica C: Superconductivity, vol. 469, no. 9-12, pp. 326-331, 2009.

[7] M. J. Pitcher, D. R. Parker, P. Adamson et al., "Structure and superconductivity of LiFeAs," Chemical Communications, vol. 45, p. 5918, 2008.

[8] J. H. Tapp, Z. J. Tang, B. Lv et al., "LiFeAs: an intrinsic FeAsbased superconductor with $T_{\mathrm{c}}=18 \mathrm{~K}$," Physical Review B, vol. 78, no. 6, p. 060505, 2008.

[9] X. C. Wang, Q. Q. Liu, Y. X. Lv et al., “The superconductivity at $18 \mathrm{~K}$ in LiFeAs system," Solid State Communications, vol. 148, no. 11-12, p. 538, 2008.

[10] D. R. Parker, M. J. Pitcher, P. J. Baker et al., "Structure, antiferromagnetism and superconductivity of the layered iron arsenide NaFeAs," Cheminform, no. 40, p. 2189, 2009.

[11] R. Mahesh, M. Rajagopalan, and B. Palanivel, "Doping effect on electronic band structure and magnetic properties of MFeAs ( $\mathrm{M}=\mathrm{Li}, \mathrm{Na})$," International Journal of Computational Materials Science and Engineering, vol. 4, no. 1, p. 1550005, 2015.

[12] I. R. Shein and A. L. Ivanovskii, "Elastic properties of singleand polycrystalline $\mathrm{LaFeAsO}, \mathrm{SrFe} 2 \mathrm{As} 2$, and $\mathrm{LiFeAs}$ basic phases for new FeAs superconductors," Technical Physics Letters, vol. 35, no. 10, pp. 961-963, 2009.

[13] Z. Li, J. S. Tse, and C. Q. Jin, "Crystal, spin, and electronic structure of the superconductor LiFeAs," Physical Review B, vol. 80, no. 9, p. 092503, 2009.

[14] P. Ravindran, L. Fast, P. A. Korzhavyi, B. Johansson, and J. Appl, "Density functional theory for calculation of elastic properties of orthorhombic crystals: application to TiSi2," Journal of Applied Physics, vol. 84, pp. 4891-4904, 1998.

[15] P. E. Blöchl, "Projector augmented-wave method," Physical Review B, vol. 50, no. 24, pp. 17953-17979, 1994

[16] G. Kresse and D. Joubert, "From ultrasoft pseudopotentials to the projector augmented-wave method," Physical Review B, vol. 59, no. 3, pp. 1758-1775, 1999.

[17] G. Kresse and J. Hafner, "Ab initio molecular dynamics for open-shell transition metals," Physical Review B, vol. 48, no. 17 , pp. 13115-13118, 1993.

[18] G. Kresse and J. Furthmller, "Efficiency of ab-initio total energy calculations for metals and semiconductors using $\mathrm{s}$ plane-wave basis set," Computational Materials Science, vol. 6, p. 15, 1996. 
[19] G. Kresse and J. Furthmller, "Efficient iterative schemes for ab initio total-energy calculations using a plane-wave basis set," Physical Review B, vol. 54, no. 16, pp. 11169-11186, 1996.

[20] J. P. Perdew, K. Burke, and M. Ernzerhof, "Generalized gradient approximation made simple," Physical Review Letters, vol. 77, no. 18 , pp. $3865-3868,1996$.

[21] J. P. Perdew, K. Burke, and M. Ernzerhof, "Generalized gradient approximation made simple," Physical Review Letters, vol. 78, no. 7, p. 1396, 1996.

[22] H. J. Monkhorst and J. D. Pack, "Special points for Brillouinzone integrations," Physical Review B, vol. 13, no. 12, pp. 51885198, 1976.

[23] R. Hill, "The elastic behaviour of a crystalline aggregate," Proceedings of the Physical Society. Section A, vol. 65, no. 5, pp. 349-354, 1953.

[24] W. Voigt, Lehrbuch der Kristallphysik, Leipzig, Taubner, Springer, Berlin, Germany, 1928.

[25] A. Reuss and Z. Angew, "Calculation of the yield strength of mixed crystals due to the plasticity condition for single crystals," Journal of Applied Mathematics and Mechanics, vol. 9, no. 1, pp. 49-58, 1929.

[26] Z. J. Chen, G. B. Xu, J. G. Yan, Z. Kuang, T. H. Chen, and D. H. Li, "First-principles investigation of mechanical and electronic properties of MFeAs $(\mathrm{M}=\mathrm{Cu}, \mathrm{Li}$, and $\mathrm{Na})$," Journal of Applied Physics, vol. 120, no. 23, p. 235103, 2016.

[27] F. Mouhat and F. X. Coudert, "Necessary and sufficient elastic stability conditions in various crystal systems," Physical Review B, vol. 90, no. 22, p. 224104, 2014.

[28] H. C. Zhai, X. F. Li, and J. Y. Du, "First-principles calculations on elasticity and anisotropy of tetragonal tungsten dinitride under pressure," Materials Transactions, vol. 53, no. 7, pp. 12471251, 2012.

[29] D. C. Wallace, Thermodynamics of Crystals, Wiley, New York, NY, USA, 1972.

[30] Q. Q. Liu, X. H. Yu, X. C. Wang et al., "Pressure-induced isostructural phase transition and correlation of FeAs coordination with the superconducting properties of 111-Type $\mathrm{Na}_{1-x} \mathrm{FeAs}$," Journal of the American Chemical Society, vol. 133, no. 20, pp. 7892-7896, 2011.

[31] X. X. Zhang, Y. C. Wang, and Y. M. Ma, "High pressure structures of "111" type iron-based superconductors predicted from first-principles," Physical Chemistry Chemical Physics, vol. 14, no. 43, p. 15029, 2012.

[32] S. F. Pugh, "XCII. Relations between the elastic moduli and the plastic properties of polycrystalline pure metals," Philosophical Magazine and Journal of Science, vol. 45, no. 367, pp. 823-843, 1954.

[33] M. Mito, M. J. Pitcher, W. Crichton et al., "Response of superconductivity and crystal structure of LiFeAs to hydrostatic pressure," Journal of the American Chemical Society, vol. 131, no. 8, pp. 2986-2992, 2009.

[34] D. Pettifor, "Theoretical predictions of structure and related properties of intermetallics," Materials Science and Technology, vol. 8, no. 4, pp. 345-349, 1992.

[35] D. M. Teter, "Computational alchemy: the search for new superhard materials," MRS Bulletin, vol. 23, no. 1, pp. 22-27, 1998.

[36] X. Q. Chen, H. Y. Niu, D. Z. Li, and Y. Y. Li, "Modeling hardness of polycrystalline materials and bulk metallic glasses," Intermetallics, vol. 19, no. 9, pp. 1275-1281, 2011.

[37] X. Q. Chen, H. Y. Niu, C. Franchini, D. Z. Li, and Y. Y. Li, "Hardness of T-carbon: density functional theory calculations," Physical Review B, vol. 84, no. 12, p. 121405, 2011.
[38] I. A. Nekrasov, Z. V. Pchelkina, and M. V. Sadovskii, "Electronic structure of new LiFeAs high-Tc superconductor," JETP Letters, vol. 88, no. 8, pp. 543-545, 2008.

[39] D. J. Singh, "Scenarios for generalized synchronization with chaotic driving," Physical Review B, vol. 78, no. 2, p. 094511, 2008. 


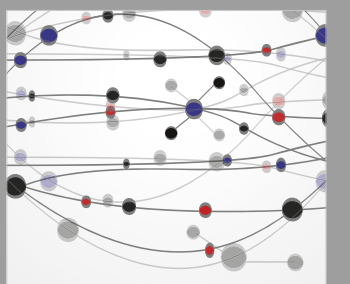

The Scientific World Journal
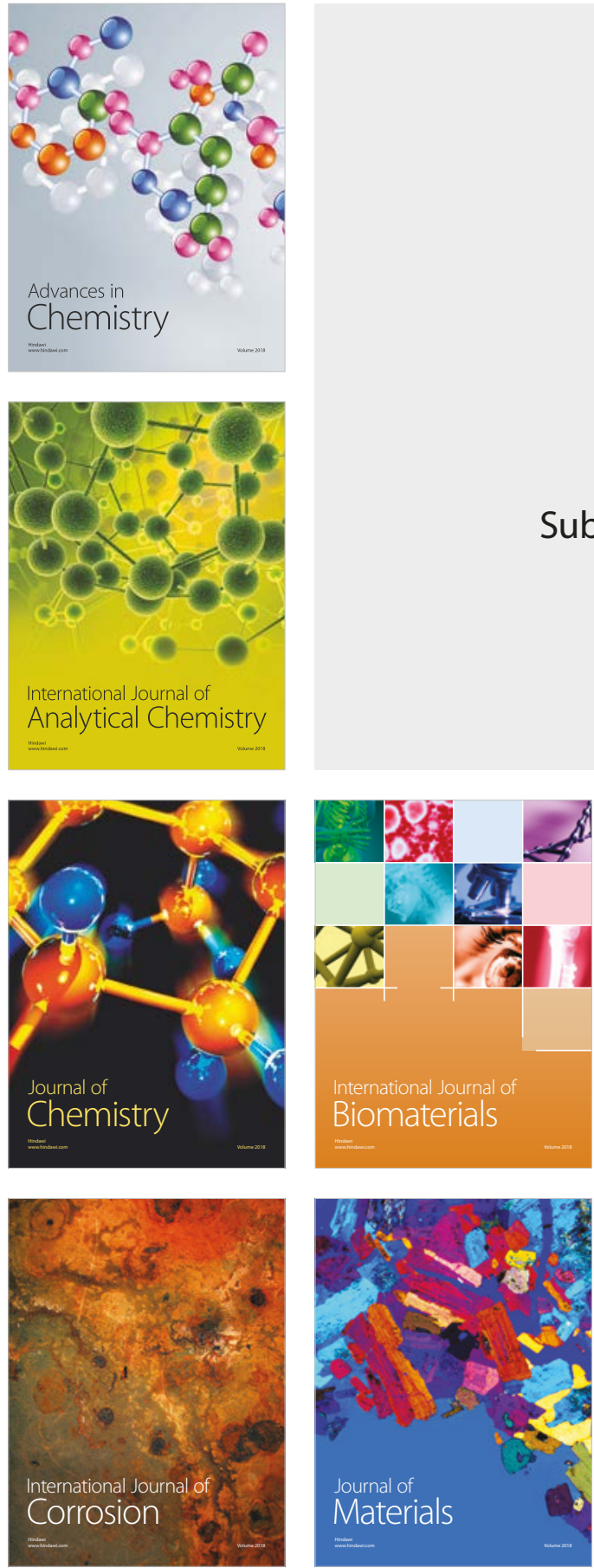

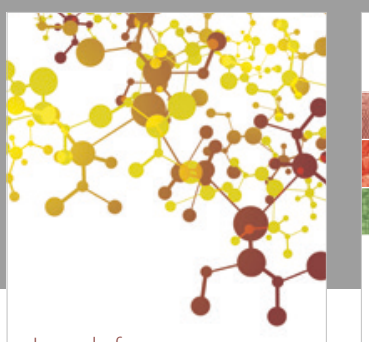

Journal of

Applied Chemistry
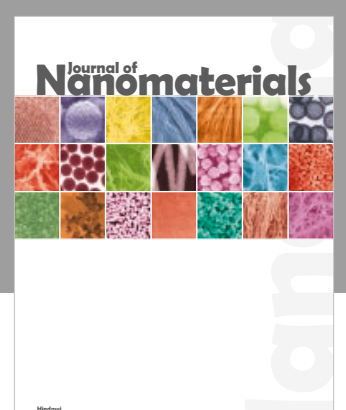

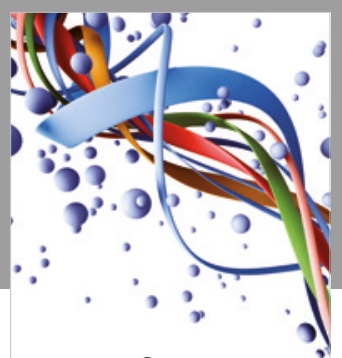

Scientifica

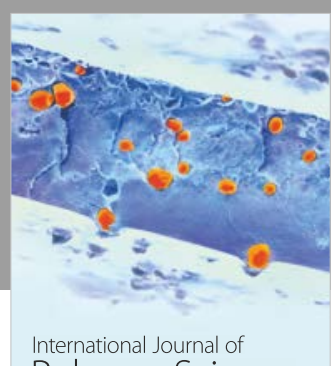

Polymer Science

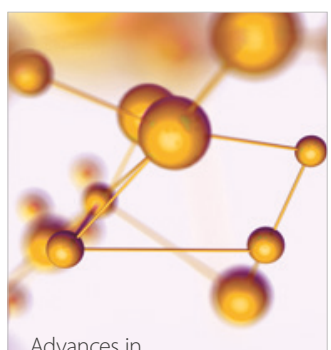

Physical Chemistry
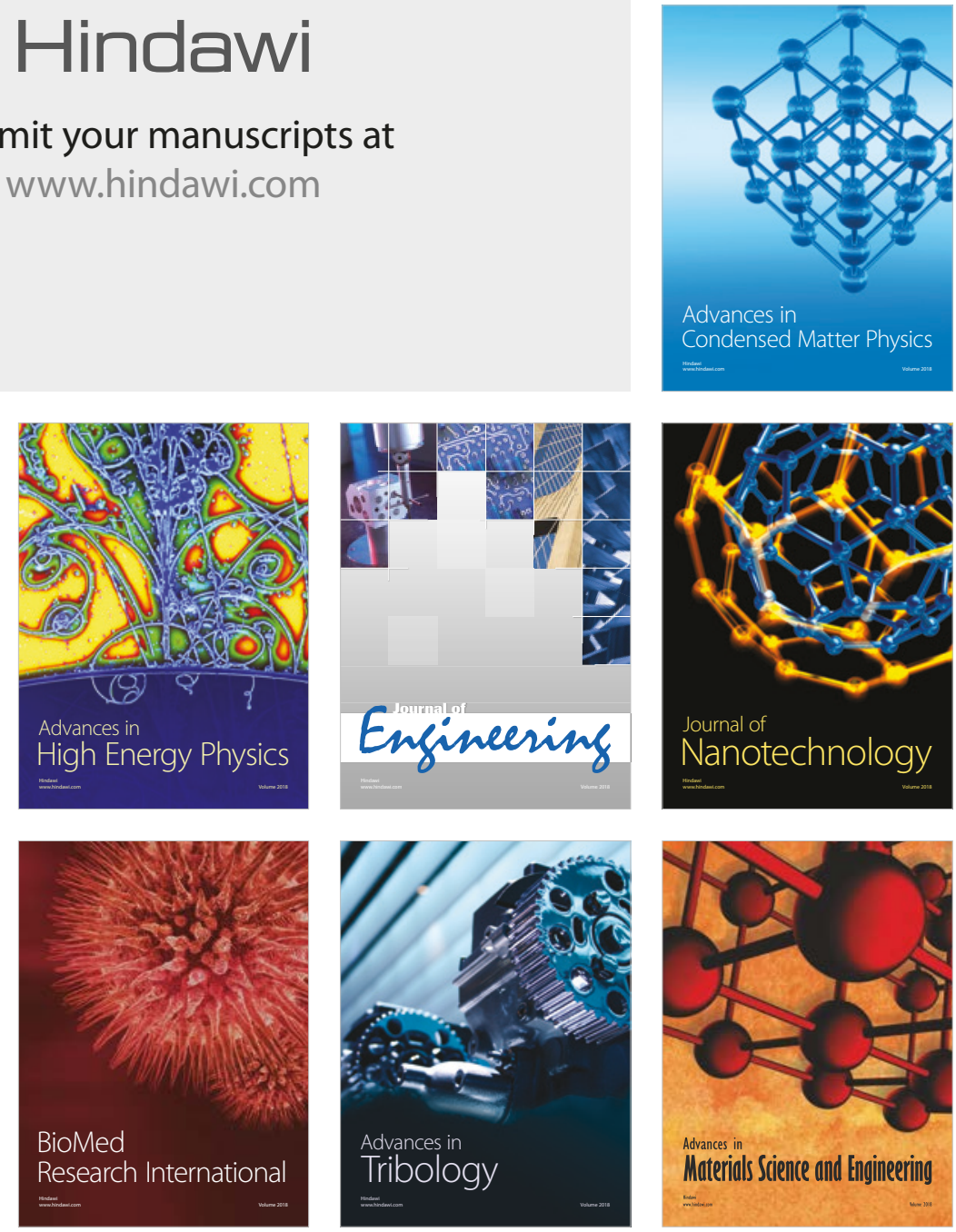